\title{
Analysis and Measurement of Session Setup Delay and Jitter in VoWLAN Using Composite Metrics
}

\author{
Muslim Elkotob \\ Division of Computer Networking \\ Luleå University of Technology \\ SE-971 87 Luleå, Sweden \\ +46920491594 \\ elkotob@ltu.se
}

\author{
Karl Andersson \\ Division of Mobile Networking and Computing \\ Luleå University of Technology \\ SE-931 87 Skellefteå, Sweden \\ +46910585364 \\ karl.andersson@ltu.se
}

\begin{abstract}
In this paper we look at the signaling for session mobility using the Session Initiation Protocol (SIP) and the Session Description Protocol (SDP). Bearing in mind that perceived voice quality is sensitive mostly to delay variation (also called jitter), we work on bounding that value for providing a better voice Quality of Service over Wireless LAN (WLAN). For mobility we use a hybrid SIP-MIP stack. The basic idea is to combine the strengths of MIP for doing fast handovers and the strengths of SIP for powerful session adaptation capabilities. Numerical calculation figures as well as real implementation results are provided. It can be observed that the session update cycle during mobility can be modified in such a way that session delay variation bounds achieved are quite low and there are almost no spikes. We use composite performance metrics that we defined in previous work to analyze the correlation between the data patterns for session signaling delay in the classical case and our proposed case to better highlight the achieved performance delta.
\end{abstract}

\section{Categories and Subject Descriptors}

C.2.6 [Computer-Communication Networks]: Internetworking.

\section{General Terms}

Algorithms, Performance, Design, Experimentation.

\section{Keywords}

Session initiation protocol, session description protocol, quality of service, wireless LAN, IP mobility.

\section{INTRODUCTION AND MOTIVATION}

Voice is sensitive to jitter more than to any other network Quality of Service (QoS) parameter. Especially in wireless networks that are multi-hop or that involve small or medium-sized cells (e.g. IEEE 802.11 networks), where the link is more error prone and

Permission to make digital or hard copies of all or part of this work for personal or classroom use is granted without fee provided that copies are not made or distributed for profit or commercial advantage and that copies bear this notice and the full citation on the first page. To copy otherwise, or republish, to post on servers or to redistribute to lists, requires prior specific permission and/or a fee.

MUM'08, December 3-5, 2008, Umeå, Sweden.

Copyright 2008 ACM 978-1-60558-192-7/08/0012 ..\$5.00. transmission is more difficult to manage and coordinate, voice traffic faces a major challenge. Voice over Wireless LAN (VoWLAN) and how to improve or provision its quality has recently become an important research issue.

For simply nomadic users, the quality of VoWLAN depends on the service level delivered by the visited access point. The performance of that particular access point then depends on the operator or service provider to whom it belongs and also on the load it experiences. For mobility scenarios however, where the user roams in 802.11 open access networks [25], there is a frequent handover taking place using Mobile IP (MIP) and session adaptation at OSI/ISO Layer 5 using the SIP protocol. We provide some insights on using hybrid SIP-MIP mobility in Section 3 of this paper.

Now for a mobile pedestrian user, having an average speed of 4-5 $\mathrm{km} / \mathrm{hr}$ or $1.3 \mathrm{~m} / \mathrm{sec}$, he would traverse a distance of 50 meters in 38 seconds and a distance of $100 \mathrm{~m}$ in 76 seconds.

There is plenty of time for a mobile terminal to perform a handoff from one access point to another. However, for a vehicular speed of $50 \mathrm{~km} / \mathrm{h}$ or $13.9 \mathrm{~m} / \mathrm{s}$, an access point coverage range of 50 meters would be traversed in about 3.6 seconds and one of about $100 \mathrm{~m}$ in approximately 7.2 seconds. So the point is that the higher the mobility speed, the harder it is to perform a handoff and the less time it is desirable that a session setup or update consumes on the radio link. Real-time applications in particular have strict requirements on network QoS parameters.

To solve the challenges posed by the problem in question, it is necessary to design an architecture that enables different signaling paths for improved session management. Furthermore, it has to be ensured that all parameters needed to build or update a multimedia session (in this case voice) are available in real-time. This is shown in Section IV of the paper.

Session setup time in multimedia using e.g. SIP is affected by the quality of the used link, and SIP uses a retransmission timer that doubles in size upon the occurrence of errors [6]. Therefore, it makes sense to model session signaling in such a way that the overall setup or update time is smaller, reducing the probability of an error occurring. 
This paper is organized as follows. After this introductory section, Section 2 analyzes related work in the area. Then Section 3 discusses multimedia session setup identifying where there is room for improving performance. Section 4 shows a high-level architecture of Open Access Networks (OAN) based on WLAN, and then explains the improved signaling scheme that is used to bound jitter for voice traffic. Section 5 then analyzes the results of the newly proposed scheme quantitatively and finally Section 6 concludes the paper.

\section{RELATED WORK}

A wide range of papers has been analyzed in parallel to conducting our work. In fact, we observed that as SIP is adopted in various access technologies, it faces a challenge in each access technology it is deployed in. VoIP over $3 \mathrm{G}$ is thoroughly discussed by Prasad et al in [6]. The authors analyze the different phases in setting up a SIP-based voice session and explore the different cases via numerical analysis in order to obtain an estimate on what it would cost in terms of time and resources to establish an appropriate VoWLAN or video-over-WLAN session in $3 \mathrm{G}$ networks.

The authors in [13] explore statistical multiplexing and try to suppress the large overhead in VoWLAN. They perform voice multiplexing using a polling mechanism in the contention-free period and a deterministic priority access for voice traffic in the contention period. They also work on reducing the overhead for voice traffic.

In [14] McGovern et al. address the problem of link adaptation using media codecs in WLAN. Different codecs are believed to result in different levels of congestion, and thus the system the authors propose switches back and forth between codecs to reduce the measured or perceived congestion level.

Bacciu et al. [12] presents a fuzzy approach to determine a soft admission control mechanism for Voice-over-IP services over Wireless LANs. A framework is defined where the provider may express the network status and the client their preferences by the means of an approach based on Fuzzy Set Theory.

Brännström el al. [4] proposes a mobility support system integrating the benefits of application-layer SIP mobility with network-layer MIP mobility where a cross-layer information system exchanges context for mobility adaptation.

Åhlund et al. [31] proposes a Running Variance Metric performance metric of wireless local area network used to predict relative traffic loads of available access points at the network layer.

The different approaches in this section either take a step in introducing combined metrics in mobility scenarios or try to solve the congestion and bad coupling of voice traffic and IEEE 802.11. We move on in the next sections to show how hybrid SIP-MIP mobility is used on a single stack with access point controllers brokering for airtime resources after every handover, and then measure the system performance. Assessing system performance in the case of SIP classical session negotiation over MIP, and then with parameter injection [3] using specialized limited signaling and more intensive computations, allows us to show a delta in performance. The key that enabled our quantitatively improved result is the architecture with proper placement of components, and specialized signaling that lead to lower session update delays and thus lower errors and shorter occupation of the airtime needed for VoWLAN session initiation or resumption.

\section{HYBRID MIP-SIP MOBILITY MECHANISMS}

The concept of hybrid Session Initiation Protocol and Mobile IP joint mobility scheme has been addressed in different contexts. A pioneering work in this area is by Tafazolli et al [33]. A MIP stack on top of which SIP resides can exploit the architecture as if using two superimposed worlds: the MIP world and the SIP world in a synchronized and aligned fashion.

We used a hybrid Mobile-IP and Session Initiation Protocol stack as a mobility solution for multimedia. MIP is used to perform fast handover and association to a new access point and to acquire a new IP address. Then we used with the Birdstep MIP client [29] a specially modified API that sends updates and event notifications upwards. In this way, the SIP mobility module which operates on logical layer 5 in the ISO/OSI model can then adapt a multimedia session (either voice or video) to the new network conditions and after MIP has handled the basic mobility part.

This hybrid stack has been used in different contexts [33] but not in the way we use it in this work. The basic idea is to combine the strengths of MIP for doing fast handovers and the strengths of SIP for powerful session adaptation capabilities. The reason for this is the fact that multimedia is often handled with SIP or peer protocols and WLAN environments have relatively small cells, so when traversing them and holding a VoIP call, the session update delay upon incurring handovers is a significant parameter. We analyze this value and show how real measurements of an existing prototype depict the improvement in performance.

\section{MULTIMEDIA SESSION SETUP ANALYSIS}

Nomadic mobility where a user moves from a location to another and then uses the Internet in a stationary manner is a common scenario. This is handled nowadays with the $3 \mathrm{G}$ or CDMA modems available from operators and service providers. On the other hand, WiFi grew beyond the hotspot scenario to become the main technology for open access networks (OAN) [5, 22, 25, 26]. OANs enable mobility within a so called distribution system where access points are connected e.g. via a virtual operator to provide continuous network coverage that enables the user to seamlessly roam from one AP to another.

Now bearing in mind that $802.11 \mathrm{~b}$ and $\mathrm{g}$ WiFi cells are larger than those of $802.11 \mathrm{a}$, but still relatively small, high speed mobility is hard to support. For this reason, we analyzed the cases with pedestrian speed mobility and low to moderate speed vehicular mobility, up to $50 \mathrm{~km} / \mathrm{h}$.

Pedestrian speed is for a user roaming with his terminal and traversing many $\mathrm{WiFi}$ access points in the process. Low and moderate speed vehicular motion is for some types of transportation which have speed limitations due to technical or regulatory reasons.

the first column presents mobility speed in $\mathrm{km} / \mathrm{hr}$

- the second column presents mobility speed in $\mathrm{m} / \mathrm{s}$

- the third column shows $t_{1}$, the time in seconds it takes a terminal to traverse an access point with coverage diameter 100 meters 
- the fourth column shows $t_{2}$, the time in seconds it takes a terminal to traverse an access point with coverage diameter 50 meters

Table 1. Motion speeds and average durations of stay within a WiFi access point.

\begin{tabular}{|l|l|l|l|l|}
\hline Case & $\mathbf{k m} / \mathbf{h}$ & $\mathbf{m} / \mathbf{s}$ & $\mathbf{t}_{\mathbf{1}}(\mathbf{s e c})$ & $\mathbf{t}_{\mathbf{2}}(\mathbf{s e c})$ \\
\hline Walking & 5 & 1.38 & 76.92 & 38.46 \\
\hline Riding & 15 & 4.17 & 23.98 & 11.99 \\
\hline \multirow{3}{*}{$\begin{array}{l}\text { Slow } \\
\text { Vehicular }\end{array}$} & 20 & 5.56 & 17.99 & 8.99 \\
\cline { 2 - 5 } & 25 & 6.94 & 14.41 & 7.20 \\
\cline { 2 - 5 } & 30 & 8.33 & 12.00 & 6.00 \\
\cline { 2 - 5 } & 35 & 10.29 & 10.29 & 5.14 \\
\hline \multirow{3}{*}{$\begin{array}{l}\text { Moderate } \\
\text { Vehicular }\end{array}$} & 40 & 11.11 & 9.00 & 4.50 \\
\cline { 2 - 5 } & 45 & 12.50 & 8.00 & 4.00 \\
\cline { 2 - 5 } & 50 & 13.89 & 7.20 & 3.60 \\
\hline
\end{tabular}

As Table 1 shows, there is enough time-room for a station to perform a handover, but the session update or setup delay can sometimes become comparable with the station's duration of stay within an access point if the error rate on the wireless part is too high [6].

Theoretical calculations in [6] show that the session setup delay for SIP in 3G networks used with UDP is about 4.61 seconds when using the Radio Link Protocol (RLP) and a transmission rate of $9.6 \mathrm{Kbps}$, and 2.9 seconds when using a transmission rate of $19.2 \mathrm{Kbps}$. The setup delay time for a SIP-based session is a few seconds over a narrow-band rate. For WLAN (broadband), this setup time can vary but would still be in the range of a 100-200 milliseconds as measurements show in [3].

To establish a session, the sequence: INVITE, 183 SDP, PRACK (final SDP), $200 \mathrm{OK}, 180$ RINGING, $200 \mathrm{OK}$, and ACK is used. SDP stands for session description protocol [27].

The same parameter sequence is necessary when updating a session due to mobility or upon the need to change session parameters (e.g. CODEC, resources). The only difference between a session update and a session initiation is that the former uses a SIP RE-INVITE whereas the latter uses SIP INVITE. Therefore, we make the observation that even a session update in WLAN which involves pure signaling can substantially disrupt or degrade a voice call when traversing many cells. VoWLAN (Voice over WLAN) this has to consider different signaling alternatives that reduce signaling delay and provide more bounded jitter with fewer spikes and a lower jitter on the average, yielding a much more acceptable perceived audio quality in IEEE 802.11.

Table 2. Message sizes and frame count per message.

\begin{tabular}{|l|l|l|l|l|}
\hline Messages & $\begin{array}{l}\text { Payload } \\
\text { (bytes) }\end{array}$ & $\begin{array}{l}\text { Message } \\
\text { (bytes) }\end{array}$ & $\begin{array}{l}\text { \# frames, } \\
\text { 9.6 kbps }\end{array}$ & $\begin{array}{l}\text { \# frames, } \\
\text { 19.2 kbps }\end{array}$ \\
\hline SIP INVITE & 700 & 728 & 37 & 19 \\
\hline SIP 183 & 835 & 863 & 44 & 23 \\
\hline SIP PRACK & 538 & 586 & 30 & 16 \\
\hline SIP 200 OK & 545 & 573 & 29 & 15 \\
\hline SIP 180 & 349 & 377 & 19 & 10 \\
\hline SIP ACK & 300 & 328 & 17 & 9 \\
\hline
\end{tabular}

The total payload size in bytes for a SIP session setup or update is: $700+835+538+545+349+300=3267$ bytes. This is a lot for a "lightweight" signaling protocol. Calculating the size of the total data required, which includes payload and overhead yields: $728+863+586+573+377+328=3455$ bytes.

Then it is obvious that a modified signaling scheme that spares some of the messages listed in Table 2 would significantly reduce session setup time and also the probability of errors occurring during updating the session when roaming between APs.

The higher the used rate, the fewer frames are needed to transmit the required information. As the rate in Table 2 is slightly more than doubled from case 1 to case 2 being first 9.6 and then 19.2 Kbps respectively, the number of frames needed shrinks to about a half. Therefore as the rate is increased, the number of frames decreases proportionally. WLAN links are error prone. Thus for a particular Frame Error Rate e.g. $0.01 \%$, the probability of getting an error gets smaller as the number of transmitted frames decreases. This makes session signaling delay cycles for sessions with higher rates lower.

\section{SUGGESTED ARCHITECTURE AND IMPROVEMENTS}

\subsection{QoS in Open Access Networks}

$[17,22]$ describe a self-contained resource allocation mechanism that was deployed on AP controllers in the architecture to perform rate control. Regulating the QoS budget of a station is done at an IP level. A tuple of parameters indicating the average packet size and the average packet rate is assigned to a station and updated dynamically by the QoS broker residing on each AP. So as the load on an access point increases, the broker redistributes capacity between stations.

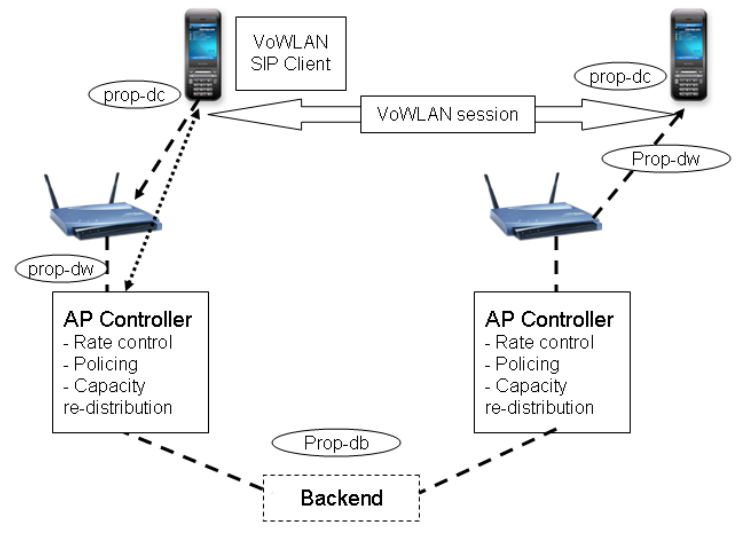

$$
\text { SIP Signaling Path }
$$

Figure 1. Basic architecture.

Now using Table1 and Figure1 we obtain Equation 1.

Equation 1. Total signaling delay.

$$
\text { delay }_{\text {total }}=2\left(\text { proc }_{d c}+\operatorname{prop}_{d w}+\operatorname{proc}_{d w}\right)+\operatorname{prop}_{d b}
$$

Figure 1 labels the various delays with symbols, so we get a one cycle delay of:

With $\operatorname{proc}_{d c}$ being the processing delay on the client, prop $p_{d w}$ being the propagation delay towards the $\mathrm{AP}, \operatorname{proc}_{d w}$ is the 
processing delay on the WLAN AC controller, and $\operatorname{prop}_{d b}$ being the propagation delay on the backend, respectively. The value delay $_{\text {total }}$ stands for one cycle of E2E delay between two clients using SIP for a VoWLAN session. The processing delay for various messages depends on the type of the message received or sent but take pretty close values.

For the classical case of negotiating session parameters either when setting up an initial session or upon updating the session settings due to mobility or resource reallocation, 6 cycles are needed, one for each of the rows in Table 2. Hence, the entire signaling cost is approximately 6 times the delay $_{\text {total }}$ variable.

In order to measure this value for a session setup, we used Java timers within our SIP-stack based on the JAIN-NIST [28] base version. The timers, which are based on threads, are started when a frame processing starts and stopped when the other party (correspondent client) finishes its processing. This way, the E2E delay in a real-implementation of the system depicted in Figure 1 is measured. Section 5 of this paper outlines some figures from the measurements made on the system.

\subsection{Experimental Setup and Signaling Scheme}

Our MIP used for the experiments used Eager Cell Switching (ECS) as an Agent Discovery and Motion Detection (ADMD) method. ECS is designed to trigger immediate handover to the new foreign agent (FA) as soon as the mobile node (MN) receives an Agent Advertisement (ADV) message from that FA. Mobile IP is used on both cases of the experimental MIP-SIP stack, so the delta achieved in delay reduction and jitter bounding is due to improved signalling in the SIP part using the parameter injection algorithm presented in [3].

As seen in the previous subsection, there is a relatively high number of messages going back and forth until a session is established in SIP. This propagation is prone to errors in WLAN causing substantial delays as calculated in [6].

Looking more closely at the RFCs in this area, such as $[7,18,19$, $21,27]$, the following branch-case can be observed as mentioned in RFC 3264 [27]: "An Offer/Answer Model Session Description Protocol": "If multiple formats are listed, it means that the offerer is capable of making use of any of those formats during the session. In other words, the answerer MAY change formats in the middle of the session, making use of any of the formats listed, without sending a new offer".

In other words, after establishing an initial session between two peers A and B engaged in a multimedia (video or voice or both) session, then when one node moves, it is allowed by RFC 3264 to modify the data stream and piggyback the new session settings with the new stream. This signaling procedure is depicted in figure 3 , the "session update" part.

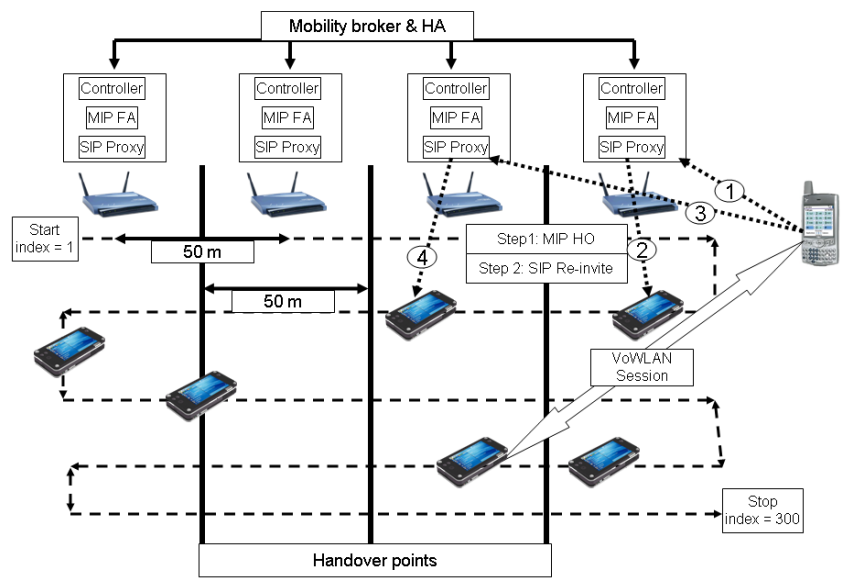

Figure 2. Experimental setup.

We used four access points placed 50 meters apart as depicted in Figure 2. Human testers were used for walking and traversing the different 802.11 b APs. Windows XP equipped laptops were used as test clients with a hybrid MIP-SIP mobility client developed jointly with Birsdstep [29]. When the signal strength of the next AP was stronger than that of the one the client was associated with, a handover was triggered (pure MIP handover). After performing the hard handover in Mobile IP and the handoff is complete, the Mobile IP API sends a trigger to activate the "REINVITE" message on the SIP part of the stack. The QoS controller on the access point assigns a new resource pair to the client upon joining that AP. Average Packet Size (APS) and Average Packet Rate (APR). Details concerning architecture, mathematical computations and implementation for airtime resources as well as new session parameters are in $[3,16]$.

After a client switches APs, MIP performs an update and then the new network parameters are fed into the SIP module via the AP QoS controller as well as upwards by the MIP API. 'The REINVITE" is launched with the newly computed parameters. For a multimedia session SIP proxy on each controller is updated with the new location (IP address) of the callee. As the user continues to move, at every handover, there is a MIP-handover followed by a SIP-SDP re-invite. The MIP handover delay is represented logically by the following time span: let the time-stamp of the last received packet (can be traced via ICMP) on the first interface be $t 1$, and the time-stamp of the first received packet on the new interface be $t 2$, then the handover cycle delay is $t 2-t 1$. SIP session signaling delay is measured via thread based timers. After the trigger from the MIP API reaches the SIP-part, a SIP-SDP session update cycle is launched. In this cycle, there is a computation part and session re-launch part with new parameters. Once the session is re-launched with a data stream obtained on the stack with updated session parameters, the timer is stopped. 


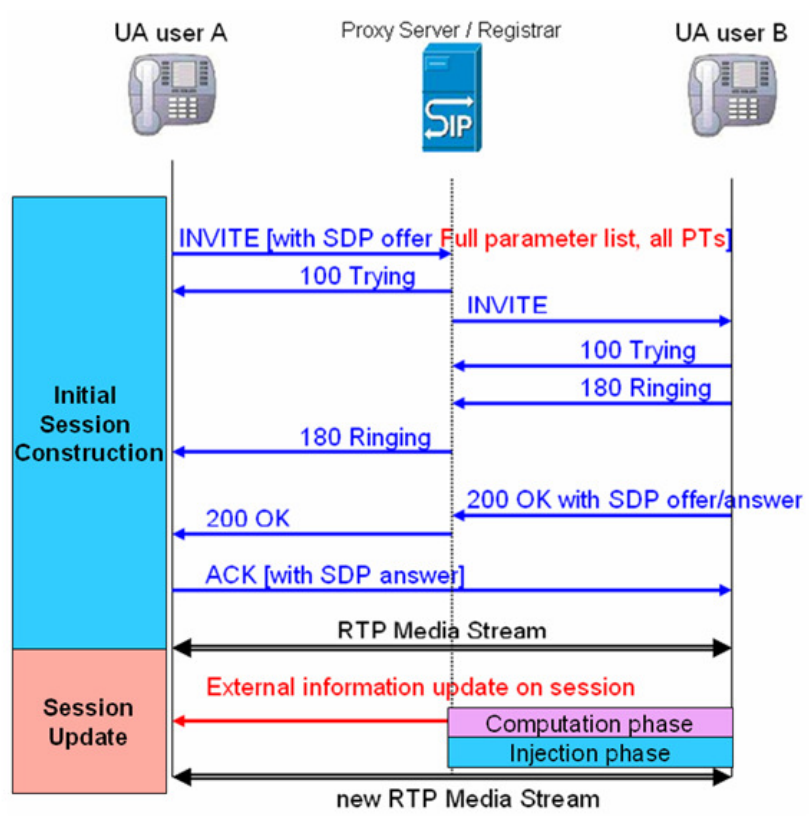

Figure 3. Modified signaling procedure.

The AP controller module that acts also as a QoS broker for roaming clients re-assigns a new rate for the client as it joins this access point [3].

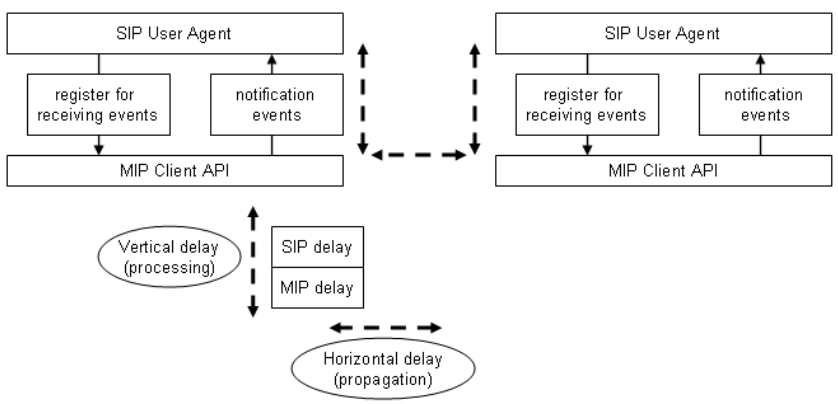

Figure 4. Delay components in the architecture used.

As Figure 4 shows, there is a delay component due to Mobile IP handovers and then on the upper part of the hybrid stack, there is the SIP delay. On each client, MIP and SIP interact with each other via an API. MIP implementation and API has been provided by Birdstep [23] and we developed the SIP stack on top for session management. After the session settings are re-computed and a new Real-time Protocol (RTP) stream is re-launched on the stack, the cycle is closed and this is the session signaling delay in SIP.

The next section will numerically compare the results for the jitter and delay variables.

\section{QUANTITATIVE ANALYSIS}

In this section, we test the two signaling cases for a SIP-session update for a voice call between two parties A and B. A is mobile, roaming from one access point to another whereas B is stationary.

In both cases the mobile nodes A and B both use a hybrid MIPSIP stack as depicted in Figure 4. The core difference between case 1 and case 2 is that in the former, the classical SIP negotiation path is used to update the session parameters of the VoWLAN call. In the latter case, the signaling path shown in Figure 3 is followed. Basically, what is done in case 2 is that: after joining a new AP during mobility, the QoS controller module attached to the AP allocates a new rate to the client. The client then recomputes the new session parameters for all multimedia sessions including the VoWLAN session [3,5]. After this computation is done, the data stream based on RTP (real-time protocol) is launched from $\mathrm{A}$ to $\mathrm{B}$ and the updated session parameters are piggybacked. Avoiding unnecessary negotiation after every time a new AP is joined reduces delay as shown in Figure 5.

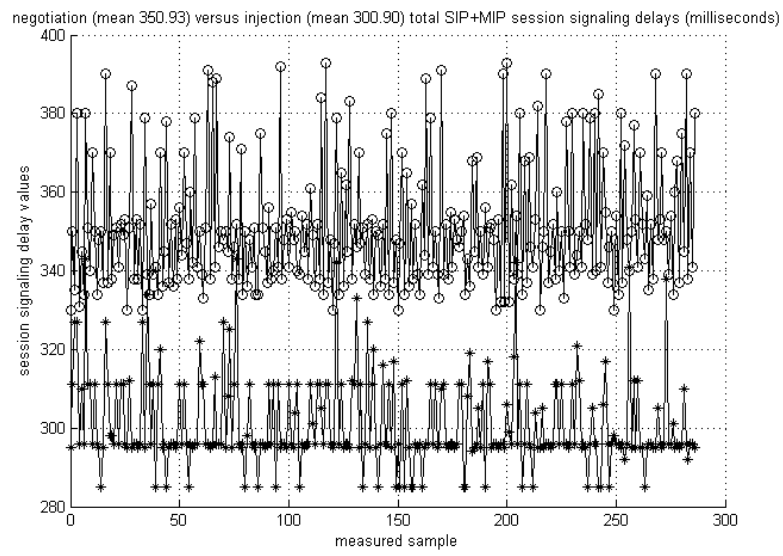

Figure 5. Session update delay times comparison for two different signaling methods.

The fact that a lower session update time is obtained in the second case that modifies the data stream avoiding negotiation automatically means that the user will experience a milder perturbation in the VoWLAN session. Perceived audio quality is furthermore more sensitive to session jitter, or delay variation. Using the delay values in Figure 5, we compute the jitter values and plot them in Figure 6.
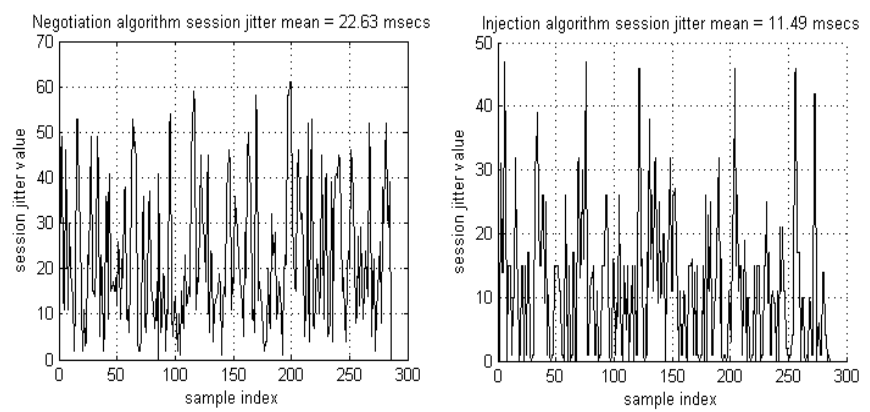

Figure 6. Jitter values for the classical and the modified signaling cases for VoWLAN.

Network QoS parameter values have been grouped into intervals based on the perceived quality by end-users. According to [15], jitter values between 0-20 milliseconds show a good performance and yield a "good" perceived audio quality. Values between 20-50 milliseconds yield an "acceptable" audio quality and values above 50 milliseconds indicate a poor performance and poor perceived voice quality.

Figure 6 depicts the jitter mean value for the different session update mechanisms. The mean value is around 23 milliseconds in the classical case and 11.5 in the modified case. As a result, using 
the classification of jitter into intervals based on the perceived audio quality, the classical signaling case falls into the "acceptable" range whereas the modified scheme which bypasses negotiation and updates the data stream for VoWLAN on each handover directly falls within the "good" range.

Using the metric: $m_{1}=$ delay $+k$; with $\mathrm{k}=5$, we obtain Figure 7 shown below. The two signaling schemes (negotiation and injection as in Figure 3) used with the hybrid SIP-MIP stack show a lot of similarity as Figure 5 and Figure 6 show. This similarity is then well reflected by the parallel curves using Metric 1 in Figure 7.

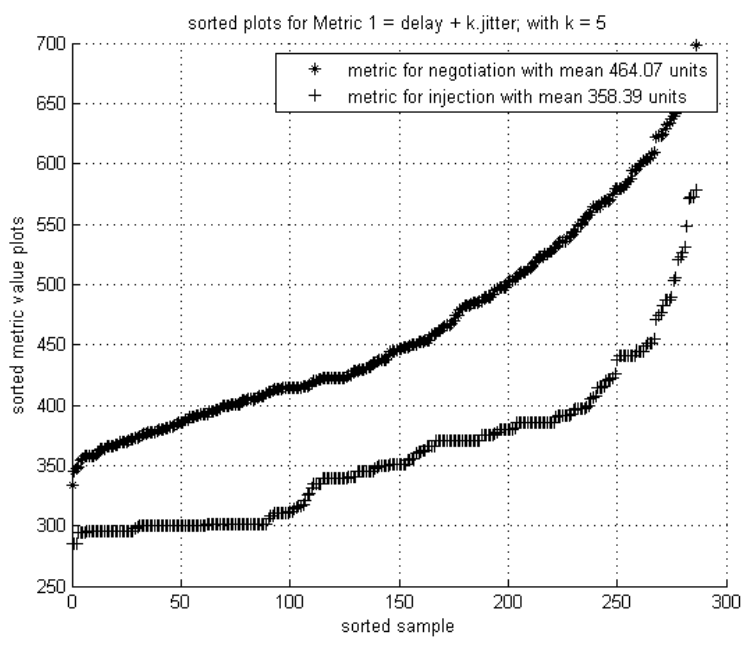

Figure 7. Metric 1 is linear in terms of delay and jitter.

Analyzing session setup and update delay cycle times in the two different cases of negotiation and injection (Figure 5), we observe from the available measured data set that:

Equation 2. Negotiation-injection linear metric relationship.

$$
\begin{aligned}
\text { delay }_{\text {negotiation }} & =k_{1} \cdot \text { delay }_{\text {injection }}+\epsilon_{1} \\
\text { jitter }_{\text {negotiation }} & =k_{2} \cdot \text { jitter }_{\text {injection }}+\epsilon_{2}
\end{aligned}
$$

Where $\mathrm{k}_{\mathrm{i}}$ is a linear constant and where all $\mathrm{k}$ values are close to each other. $\epsilon$ is a small correction variable that indicates the delta spread or variance of the data between the first and second schemes as shown in Figure 7 and Figure 8. The two above equations are also valid for the mean values of the session delays and jitters.

Equation 3: Coefficient calculation for used metrics from measured data.

$$
\begin{gathered}
k_{1}=350.93 / 300.90=1.166 \\
k_{2}=22.63 / 11.49=1.97
\end{gathered}
$$

Using the metric $m_{2}=$ delay + jitter $^{2}$ (Figure 8) shows a diversion between the curves, and does not say much about two schemes except that the injection mechanism outperforms the negotiation mechanism with hybrid mobility. The behavioral relationship is nevertheless better shown (to be a behavioral pattern and distribution similarity) is better seen using metric $m_{1}$.

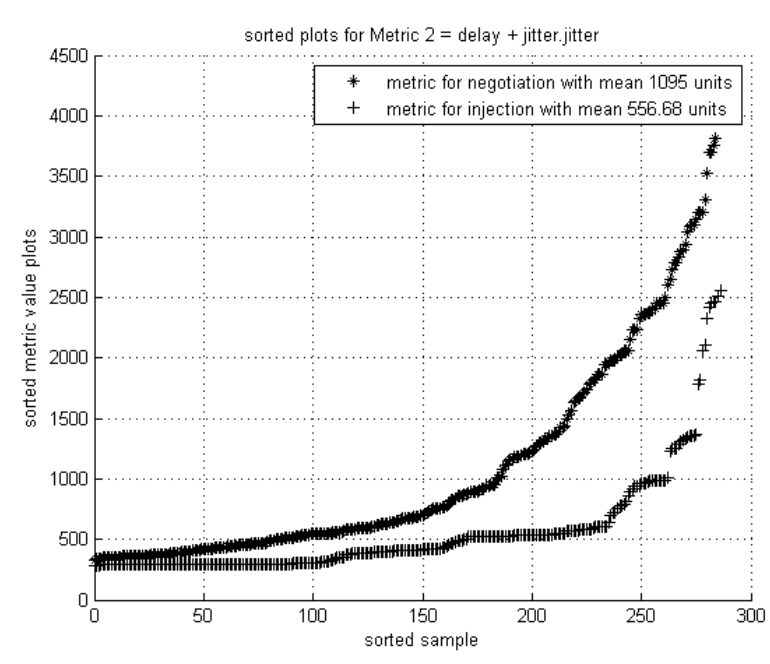

Figure 8. Metric 2 adds linear delay and quadratic jitter.

We hence see that for each particular pattern or scenario use-case, a specific composite metric is suitable. In our case $\mathrm{m} 1$ turns out to be more suitable then $m_{2}$ due to the resemblance and correlation between the two data patterns for session setup delay.

\section{CONCLUSION AND FUTURE WORK}

We have shown that it is possible to use a different signaling mechanism in open access WLAN-based networks for a better performance. When replacing classical P2P negotiation via SIP messages with resource control at the access point, all the mobile stations needs to do is to re-compute their session resources after a handover and then launch an updated stream. This technique yields lower delay values and more strongly bounded jitter. The key issue here is fewer bytes and messages for session setup or update in the parameter injection scheme compared to negotiation. Jitter reduction from around 23 milliseconds on the average down to around 11.5 milliseconds yields a much better perceived audio quality for VoWLAN. Optimizing session setup delay cycle times and the variation of those cycle times (what we call session jitter) contributes to stabilizing voice-over-WLAN and makes it an attractive application and service for virtual operators and service providers. Furthermore, the metrics used in our previous publications allow us to graphically convey the system behavior and pattern in session delay distribution and bounds. The demonstration was performed for a pedestrian speed experiment, but the work can be extended to low and intermediate speed vehicular scenarios.

Future work includes activities such as using more metrics e.g. by Andersson et al, Brannstrom et al, and Åhlund et al [4] that variously combine jitter and delay in order to formally represent each signaling scheme and evaluate it. In addition, scalability tests for VoWLAN can be done to see how different signaling schemes perform with increasing load.

\section{ACKNOWLEDGMENTS}

The authors would like to thank the colleagues from industrial partners especially Alcatel-Lucent, and Birdstep Technology ASA for contributing to joint modules on which our work co-depends. Furthermore, thanks to the valuable expert reviews from colleagues before final submission. 
The work presented in this article was also partly based on results from the HybriNet@Skellefteå [34] project supported by Skellefteå Kraft.

\section{REFERENCES}

[1] C.Politis, K. Chew , and R. Tafazolli, "Multilayer Mobility Management for All-IP Networks: Pure SIP vs. Hybrid SIP/Mobile IP," 57th IEEE Semiannual Vechicular Technology Conference VTC2003

[2] K. Kim and K. Nahrstedt, "A Resource Broker Model with Integrated Reservation Scheme," in Proceedings of the IEEE International Conference on Multimedia and Expo. New York, NY, August 2000

[3] M. Elkotob and S.Albayrak, "A Parameter Injection Algorithm for Real-time Traffic in 802.11 Open Access Networks", IEEE GLOBECOM 26-30 November, 2007, Washington D.C., USA, pp 2558-2564.

[4] R. Brannstrom, E. R. Kodikara, C. Ahlund, and A. Zaslavsky, "Mobility Management for Multiple Diverse Applications in Heterogeneous Wireless Networks", IEEE CCNC 2006, pp 818-822

[5] M. Elkotob, H. Almus, S. Albayrak, and K. Rebensburg: The Open Access Network Architectural Paradigm Viewed Versus Peer Approaches; Telektronikk Journal for Telecommunications, Volume 102 No. 3-4-2006; ISSN: 0085-7130

[6] H. Fathi, S. Chakraborty, and R. Prasad, "Optimization of SIP Session Setup Delay for VoIP in 3G Wireless Networks", IEEE Transactions on Mobile Computing Vol. 5, No. 9, September 2006, pp 1121-1132

[7] Rosenberg, J., Peterson, J., Schulzrinne, H. and G. Camarillo, "Best Current Practices for Third Party Call Control (3pcc) in the Session Initiation Protocol (SIP)", RFC 3725, March 2004.

[8] S. Li, "Research challenges and perspectives of voice over wireless" Emerging Information Technology Conference, 2005. 15-16 Aug. 2005, IEEE Digital Library DOI: 10.1109/EITC.2005.1544362

[9] W. Quan and D. Ming Hui, "Queueing analysis and delay mitigation in the access point of VoWLAN" IEEE International Symposium on Communications and Information Technology, 2005.

Volume 2, 12-14 Oct. 2005 Page(s):1160 - 1163

[10] Xuemin Shen; Yi-Bing Lin; Ai-Chung Pang; Jianping Pan , "Voice over wireless local area network"; Wireless Communications, IEEE Volume 13, Issue 1, Feb. 2006 Page(s):4 - 5

[11] Koga, H.; Kashihara, S.; Fukuda, Y.; Iida, K.; Oie, Y , “A quality-aware VoWLAN architecture and its quantitative evaluations", IEEE Wireless Communications, Volume 13, Issue 1, Feb. 2006 Page(s):52 - 59

[12] Bacciu, D.; Botta, A.; Badia, L., "Fuzzy Admission Control with Similarity Evaluation for VoWLAN with QoS Support", Fifth Annual Conference on Wireless on Demand Network Systems and Services, 23-25 Jan. 2008. WONS 2008, pp 57 $-64$
[13] Hai Jiang and Weihua Zhuang, "Performance Enhancement for WLAN Supporting Integrated Voice/Data Traffic", IEEE Conference on Communications, ICC 2006, Volume 8, June 2006 Page(s):3808 - 3813

[14] McGovern, Philip; Murphy, Sean; Murphy, Liam, "Addressing the Link Adaptation Problem for VoWLAN using Codec Adaptation", IEEE Global Telecommunications Conference, November 2006. GLOBECOM '06

[15] P. Calyam and C. Lee: Characterizing Voice and Video Traffic Behavior over the Internet: International Symposium on Computer and Information Sciences (ISCIS). Proceedings published by Imperial College Press in a special edition of "Advances in Computer Science and Engineering" Book Series, 2005

[16] F.J.M. Panken, G.J.Hoekstra: Multi-service traffic profiles to realize and maintain QoS guarantees in wireless LANs, submitted for possible publication in IEEE Wireless.

[17] Frans Panken, Gerard Hoekstra, Sietse van der Gaast: Resource allocation and guarantees for real-time applications in WLANs, Telektronikk, Telenor's journal of technology, Volume 102 No. 3/4 2006; ISSN 0085-7130

[18] RFC 3551: RTP Profile for Audio and Video Conferences with Minimal Control; H. Schulzrinne and S. Casner, July 2003 [Obsoletes RFC 1890]

[19] RFC 3312: Integration of Resource Management and Session Initiation Protocol (SIP); G. Camarillo, W. Marshall, and J. Rosenberg; October 2002

[20] IEEE 802.11 official group site: grouper.ieee.org/groups/802/11/

[21] RFC 3261: SIP: Session Initiation Protocol, by Rosenberg, Schluzrinne, Camarillo, Johnston, Peterson, Sparks, Handley, and Schooler; June 2002

[22] Panken, F.; Hoekstra, G.; Barankanira, D.; Francis, C.; Schwendener, R.; Grondalen, O.; Jaatun, M.G , "Extending 3G/WiMAX Networks and Services through Residential Access Capacity [Wireless Broadband Access]" IEEE Communications Magazine, Volume 45, Issue 12, December 2007 Page(s):62 - 69

[23] Birdstep Technology: www.birdstep.com

[24] Q. Wang and M. A. Abu-Rgheff, "Integrated Mobile IP and SIP Approach for Advanced Location Management," Proc. IEEE 4th International Conference on 3G Mobile Communication Technologies 3G2003, June 2003.

[25] John Charles Francis and Christian Fischer, "Mobile Networks Beyond 3G", Comtec 6/2003

[26] F. Panken, M. G. Jaatun, H. Bryhni, P. Engelstad, L. Hansson, G. Hoekstra, and T. H. Johannessen, "Architecture for Sharing Residential Access with Roaming WLAN Users", Telektronikk Journal for Telecommunications, Q3-4 2006, pp 48-59.

[27] RFC 3264: An Offer/Answer Model with the Session Description Protocol (SDP), Rosenberg and Schulzrinne

[28] JAIN-NIST Java VoIP project: http://snad.ncsl.nist.gov/proj/iptel/ 
[29] Birdstep Mobile IP Client: http://www.birdstep.com/Products/Secgo-Products/MobileIP-Toolkit/

[30] Fuzzy Admission Control with Similarity Evaluation for VoWLAN with QoS Support Davide Bacciu, Alessio Botta, Leonardo Badia IMT Lucca Institute for Advanced Studies Piazza S. Ponziano 6, 55100 Lucca, Italy

[31] Running Variance Metric for evaluating performance of Wireless IP Networks in the MobileCity Testbed, Christer Åhlund, Robert Brännström, and Arkady Zaslavsky; First International Conference on Testbeds and Research Infrastructures for the Development of Networks and
Communities, 2005. IEEE Tridentcom 23-25 Feb. 2005 Page(s): 120 - 127

[32] K. Andersson and C. Åhlund, An architecture for seamless mobility management in various types of applications using a combination of MIP and SIP. In Proceedings of the 4th Swedish National Computer Networking Workshop (SNCNW 2006), Luleå, Sweden, October 2006

[33] Multilayer Mobility Management for All-IP Networks: Pure SIP vs. Hybrid SIP-Mobile IP: Christos Politis, Kar Ann Chew, Rahim Tafazolli; In proceedings of the 57th IEEE Semiannual Vehicular Technology Conference, 2003 Volume 4, 22-25 Page(s):2500 - 2504 vol.4

[34] Hybrinet Project: www.hybrinet.org 